\title{
Vitamin D in ocular and systemic health
}

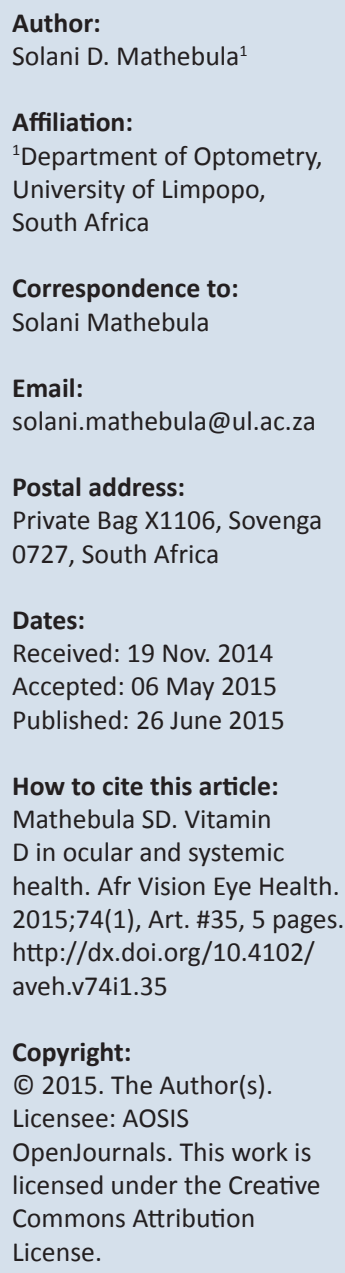

Accumulated data supports the argument that vitamin D possesses several biological and molecular actions apart from its role in calcium absorption and facilitation of gene expression. Vitamin D deficiency has been an associated risk factor for cardiovascular disease, metabolic syndrome and ocular complications. The aim of this review is to summarise the most relevant data regarding these associations and to try to clarify whether, and to what extent, oral vitamin D supplementation could be used as a beneficial intervention in such diseases.

Vitamin D is produced in skin exposed to sunlight UVB radiation and is then metabolised by the kidney into calciferol, which is an active form. The main function of vitamin D is to promote calcium and phosphorus absorption, and studies have shown that a lack of it plays an important role in ocular conditions, such as age-related macular degeneration and diabetic retinopathy. Recent studies have suggested that vitamin D may protect the diabetic retina; however, other vitamin D-associated conditions (diabetes, hypertension and cardiovascular diseases) may result in secondary ocular manifestations and the potential for sight-threatening complications. The purpose of this review is to describe the current literature on the role of vitamin D in ocular and systemic wellness. However, more research is needed to determine if increasing levels of this vitamin can assist in preventing age-related macular degeneration or diabetic retinopathy. Since vitamin D is a circulating steroid hormone, its receptors are found in almost every cell in the human body, and this suggests that vitamin $\mathrm{D}$ might have a very broad role for overall health. However, there is still demand for further research to clarify the clinical use of vitamin $\mathrm{D}$ in the prevention and treatment of various chronic diseases.

\section{Introduction}

Sunlight exposure has always been important as an energy source in the food chain. For millions of years phytoplankton has been converting the sun's energy by photosynthesis into energyproducing sugars. ${ }^{1,2,3}$ Vitamin D (also known as the sunshine vitamin) is known to promote calcium absorption in the digestive system thus preventing rickets in children, and osteomalacia (non-specific throbbing, aching, bone pain, weakness and muscle discomfort) in adults. 1,2,3,4,5,6,7,8,9 Vitamin D deficiency and insufficiency coincide with osteoporosis (disorder characterised by low bone mineral density and increased risk of fracture). Calcium ions are essential for a large variety of cellular and metabolic processes in the body. ${ }^{9}$ The first clinical description of rickets was made in 1651 but it was only in 1922 that an association was found between lack of sunlight and rickets, and that vitamin D was capable of curing rickets. ${ }^{1}$

The purpose of this review is to enhance awareness on the potential ocular and systemic benefits of vitamin D. The identification of the vitamin D receptor in almost every human cell suggests that it may have a vital role for not only ocular but for overall health.

\section{Sources of vitamin D}

There are two forms of vitamin $\mathrm{D}$, namely vitamin $\mathrm{D}_{3}$ or cholecalciferol, which is formed in the skin after exposure to ultraviolet $B$ radiation, and vitamin $D_{2}$ or ergocalciferol, obtained by irradiation of foods or plants. ${ }^{2,4}$ The major source of vitamin D for humans is exposure of the skin to the sunlight. ${ }^{1,10}$ Excessive exposure to sunlight does not result in overproduction of vitamin $\mathrm{D}_{3}$ in the skin but is converted to other photoproducts.

Vitamin $\mathrm{D}_{2}$ is produced in yeast and plants, ${ }^{1,10}$ and the best sources of dietary vitamin $\mathrm{D}$ are cod liver oil, eggs, oily fish and UV irradiated mushroom. Juice products, bread, cereals and dairy products are fortified with vitamin D. ${ }^{1}$ The three ways to obtain vitamin D are through sunlight, food and supplementation. ${ }^{11}$ 


\section{Photosynthesis of vitamin D}

Humans get most of their vitamin D from sunlight (UVB). When a sufficient amount of light strikes the epidermis, it triggers a reaction with a substance called 7-dehydrocholesterol. This substance is needed for the formation of the previtamin $D_{3}$. This previtamin $D_{3}$ is then rapidly converted to the non-active storage form called 25 -hydroxyvitamin $\mathrm{D}_{3}$, also known as $25(\mathrm{OH}) \mathrm{D}$ or calcidiol via hydration in the liver ${ }^{4,11}$ and subsequently in the kidney to form 1,25-hydroxyvitamin $\mathrm{D}_{3}$, also known as $1,25(\mathrm{OH})_{2} \mathrm{D}$ or calcitriol., ${ }^{411}$ This 1,25-hydroxyvitamin $\mathrm{D}_{3}$ is the active metabolite or endogenous hormone, which is responsible for most of the physiological actions of vitamin D. Vitamin D is fat soluble and is stored in the body fat. Any excess vitamin D that is produced during exposure to the sunlight can be stored in body fat and used during winter when little vitamin D is produced in the skin. ${ }^{1,2,3}$

Through nutrition, vitamin D intake is limited. ${ }^{4}$ Vegetarian diets are restricted to the plant vitamin $\mathrm{D}_{2}$ that is only present in some mushrooms. Vitamin $\mathrm{D}_{3}$ is not found in food-borne plants. ${ }^{5}$

The synthesis of cutaneous vitamin D is mainly affected by the amount of solar UVB radiation reaching the human skin ${ }^{11}$, thickness of the ozone layer in the atmosphere, season of the year and time of the day. ${ }^{1,2,3,12}$ Sunscreens work by absorbing UVB and UVA radiation before it enters the skin. ${ }^{10}$ Therefore, sunscreen with a sun protection (SPF) reduces the capacity of the skin to produce vitamin $\mathrm{D}$.

\section{Ocular wellness}

The main function of vitamin D is the absorption of calcium and phosphorus, which helps form and maintain bone mineralisation. ${ }^{2,13}$ Calcium is a crucial building block of bone. Vitamin D may also play a role in preventing and treating cancer, $^{13}$ hypertension, ${ }^{1}$ diabetes, ${ }^{14,15}$ atherosclerosis and multiple sclerosis ${ }^{6}$. Most of these conditions have secondary ocular manifestations, such as age-related macular degeneration (AMD) and retinopathy.

There are several published studies suggesting an association between vitamin D deficiency and ocular complications, including AMD. In 2007, Parekh, Chappell, Millen, Albert and Mores ${ }^{16}$ conducted a cross-sectional study to evaluate the association between levels of vitamin D in serum and prevalence of AMD. Results from this study provided evidence that vitamin D may protect against AMD. The researchers further speculated that the beneficial effect of vitamin $\mathrm{D}$ might be via an anti-inflammatory effect. However, they cautioned that further investigations are needed to confirm this association. Another study has shown that vitamin $\mathrm{D}$ intake reduces $\mathrm{C}$-reactive protein, a marker of systemic inflammation. ${ }^{17}$

In another cross-sectional study, ${ }^{18}$ the relationship between serum vitamin $\mathrm{D}$ and concentrations and the prevalence of early AMD was investigated. The researchers further reiterated that high serum vitamin $\mathrm{D}$ concentrations may protect against early AMD in post-menopausal women younger than the age of 75 years. Intake of vitamin D from foods and supplements was related to decreased odds of early AMD. However, more studies are needed to better understand the potential interaction between vitamin $\mathrm{D}$ status, and genetic and lifestyle factors with respect to risk for early AMD.

Kaur et al. ${ }^{19}$ conducted another cross-sectional study to examine the hypothesis that vitamin D deficiency is associated with increased prevalence of retinopathy in young people with type 1 diabetes. This study found that the inflammatory and angiogenic effect of vitamin D deficiency may contribute to the early retinal vascular damage. Whether vitamin D supplementation in diabetic patients can prevent or improve the prognosis for retinopathy remains to be investigated.

In 2005, Seddon, Reynolds, Shah and Rosner ${ }^{20}$ reported the results of a study on the association of vitamin D intake with AMD amongst 14 monozygotic twins with discordant AMD phenotypes. The researchers found higher dietary intake of vitamin $\mathrm{D}$ in the twins with early AMD compared to co-twins with more severe AMD. Twins with low vitamin $\mathrm{D}$ intake were more exposed to the development of severe AMD compared with their co-twins with higher intake. Their findings suggested that dietary intake of vitamin D could be involved in the aetiology of AMD, in addition to genetic susceptibility. This study suggested that behavioural and nutritional factors associated with epigenetic mechanisms are involved in the aetiology of AMD. Dietary intake may contribute valuable information about the potential role of vitamin D in the pathogenesis of AMD as well as other health outcomes (hypertension, diabetes, cancer and stroke).

Vitamin D deficiency has been associated with both increased risk and severity of multiple sclerosis, as it has a modulating effect on the immune process that causes inflammation and demyelination and axonal damage. Recently, a study was conducted to determine the association of vitamin D and retinal nerve fibre layer in multiple sclerosis patients without a history of optic neuritis..$^{21}$ The results of this study showed that there was no relationship between vitamin D levels and the extent of retinal axonal loss. Mutti and Marks ${ }^{22}$ found that myopes had lower levels of blood vitamin D compared with non-myopes when adjusted for age and dietary intake. Adjusted for dietary variables, myopes appear to have lower average blood levels of vitamin $\mathrm{D}$ than non-myopes. Another study is needed to replicate this one on a larger sample.

\section{Age-related macular degeneration and cardiovascular disease}

There is evidence suggesting that AMD and cardiovascular disease (CVD) share similar risk factors and common pathogenetic mechanisms. $23,24,25$ Both AMD and CVD are linked with various cardiovascular risk factors, such 
as smoking, hypertension, inflammatory markers and common genetic variants. Both diseases are associated with atherosclerosis and inflammation, although the exact molecular pathway linking AMD and CVDremains unknown. Therefore, vitamin $\mathrm{D}$ insufficiency could be the possible cause of both AMD and CVD. Vitamin D supplementation is needed to prevent and treat these diseases. Because of its anti-inflammatory properties, vitamin D may suppress the cascade of destructive inflammation that occurs at the level of the retinal pigment epithelium-choroid interface in early stages of AMD.

Sun et al. ${ }^{25}$ found that signs of early AMD were associated with incident coronary heart events. However, the mechanisms underlying this association are not fully understood. However, a study by Fernandes et al. ${ }^{26}$ did not find any association between AMD and incidents of cardiovascular diseases. There are no general agreement about the clinical importance of AMD as a marker for stroke and cardiac-related events. ${ }^{27}$ Mascitelli et al. ${ }^{28}$ suggested that vitamin D insufficiency might play a role in the development of both AMD and coronary heart disease. Vitamin D deficiency has been proposed to be associated with a cytokine profile that favours inflammation (C-reactive protein) which could dispose to both AMD and coronary heart disease. ${ }^{17}$ There is an exponential growing interest in inflammation as one of the important potential risk factors for the development of AMD. However, the effect of vitamin D supplementation on the prevention and treatment of AMD has not yet been studied in a randomised clinical trial.

\section{Vitamin D and diabetic retinopathy}

Diabetic retinopathy (DR) is one of the most frequent complications of diabetes mellitus. Patrick, Visintainer, Shi, Weiss and Brand ${ }^{29}$ conducted a cross-sectional study to explore if any association exists between vitamin D deficiency and DR. They found that subjects with severe forms of retinopathy had greater prevalence of vitamin $\mathrm{D}$ deficiency. In a recent cross-sectional study, researchers investigated a possible association of vitamin D deficiency with DR in young Japanese type 1 diabetic patients. Vitamin D deficiency was significantly associated with retinopathy. ${ }^{30}$ Such results suggested that vitamin D deficiency may be involved with DR. Another study has suggested that vitamin D may protect the diabetic retina. ${ }^{14}$

Results from the study by Payne et al. ${ }^{31}$ found that diabetic patients have lower serum vitamin $\mathrm{D}$ levels than those without diabetes. They concluded that patients at risk for DR should consider the use of vitamin D supplements. These researchers maintained that DR may be caused, in part, by insufficient vitamin D intake. This study has also found that patients with DR, especially the proliferative form, are more likely to have insufficient serum vitamin D levels than people without diabetes, suggesting a link between vitamin D deficiency and DR. ${ }^{32}$ They excluded patients with type 1 diabetes. However, both type 1 and type 2 diabetes have been associated with low vitamin $\mathrm{D}$ status.

\section{Vitamin D and dark-skinned people}

People with naturally dark skin are at risk of vitamin D deficiency. ${ }^{33,34}$ Melanin is a large opaque cellular structure ${ }^{10}$ that gives the skin colour, and the skin increases its melanin concentration in response to sunlight. Melanin absorbs and scatters electromagnetic radiation and thus competes with 7-dehydrocholesterol for UVB photons. Dark skin protects the cells from the sun's damaging effects. ${ }^{10}$ Higher melanin levels in the skin of dark-skinned people block the action of UVB radiation on the vitamin D precursor in the skin, so dark-skinned people require much longer sun exposure to produce vitamin D and are at risk of vitamin D deficiency. Adequate vitamin $\mathrm{D}$ is required for the proper absorption of calcium, and for the immune system and neuromuscular functions. A deficiency may play a role in certain types of cancers, cardiovascular diseases, age-related macular degeneration and other infections. ${ }^{10}$

\section{Adequate levels of vitamin D}

Sun exposure alone ought to suffice for vitamin D sufficiency; however, vitamin D deficiency is prevalent despite plentiful sunshine. Lifestyle choices such as clothing (that may limit sun exposure) or vegetarianism (which may limit vitamin D-rich dietary options) may be the causes of vitamin D deficiency. ${ }^{35,36,37}$ Vitamin D sufficiency is defined as serum levels of 25-hydroxyvitamin D or 25(OH)D of $30 \mathrm{ng} / \mathrm{mL}-$ $100 \mathrm{ng} / \mathrm{mL}$, whilst the levels of vitamin D insufficiency are in the range of $21 \mathrm{ng} / \mathrm{mL}-29 \mathrm{ng} / \mathrm{mL} .^{35}$ The levels of vitamin D deficiency are below $20 \mathrm{ng} / \mathrm{mL}^{35}$

Without adequate levels of 1,25-dihydroxyvitamin D in the bloodstream, dietary calcium cannot be absorbed. ${ }^{35}$ Low calcium levels lead to an increase in serum parathyroid hormone concentration, which leads to increased tubular reclamation of calcium in kidneys and reabsorption from the skeletal at the cost of lowering bone density. ${ }^{36}$ This would lead to weakened and brittle bones that break easily. There is currently no optimum level of vitamin D that people really should be receiving, but enough is needed to build healthy bones and teeth.

\section{Who is at risk of developing vitamin D deficiency?}

People who are not regularly exposed to the sun, persons over 60 years of age, those with dark skins, persons affected by conditions which inhibit vitamin D metabolism and storage, obese persons, infants born to mothers with low vitamin D and those living in regions with low sunlight levels. . $2,3,4^{2}$ Vitamin D deficiency may cause no symptoms at all or there could be non-specific symptoms, such as headache or aches and pains in bones and muscles. ${ }^{2,3}$ Hypovitaminosis $\mathrm{D}$ can be attributed to lifestyle and environmental factors that reduce exposure to sunlight, which is required for UVB-induced vitamin D production in the skin. ${ }^{37,38,39}$ Several conditions that can cause vitamin $\mathrm{D}$ deficiency include poor dietary intake of vitamin $\mathrm{D}$, inadequate sun exposure, malabsorption 
due to inflammatory bowel disease, gastric surgery and a variety of medications such as anticonvulsants, long-term use of glucocorticoids, antifungal and HIV and/or AIDS medications. ${ }^{38,39}$ Measures to prevent vitamin D deficiency include increased skin exposure to sunlight for short periods, increased fortification of food items with vitamin D and vitamin D supplementation.

\section{Effect of vitamin D excess}

In the absence of adequate exposure to sunlight, at least 1000 IU of dietary or supplemental vitamin D intake is required daily to prevent vitamin D deficiency. Vitamin D from sunshine cannot be overdosed but taking supplements is not risk-free. Ingesting too much vitamin D (doses that exceed $10000 \mathrm{IU})$ can cause high blood calcium levels (hypercalcemia or hypercalcuria), which can damage the kidneys. Hypercalcemia includes thirst, itchiness, diarrhoea, malaise, wasting, polyuria and diminished appetite. The tolerable upper level for daily vitamin D intake is $4000 \mathrm{IU}$. There is little or no documented case of vitamin D intoxication due to excess exposure to sunlight. ${ }^{40,41,42,43}$ However, long-term excessive exposure and repeated sunburns are associated with non-melanoma skin cancers. ${ }^{10}$ Hypervitaminosis D was reported to have been caused by drinking milk, which had been incorrectly and excessively fortified with vitamin $\mathrm{D}$, or when treatment was performed with an active metabolite. ${ }^{41,43}$

\section{Vitamin D, sunlight and skin cancer}

Chronic excessive sun exposure damages the elastic structure of the skin. It also causes skin cancer and wrinkling. The most serious form of skin cancer is melanoma. However, most melanomas occur even on non-sun exposed areas. Exposure to sunlight for 5-15 minutes between the hours of 10:00 and 15:00 during spring, summer and autumn is enough to produce adequate levels of vitamin D. ${ }^{1,2,3,4,10}$ Patients should avoid extended periods of unprotected midday sunshine. Intentional sun exposure for any length of time is not beneficial. Sufficient vitamin D level may be achieved with limited sun exposure.

\section{Conclusion}

Vitamin D deficiency is probably the most under-diagnosed and under-treated nutritional deficiency. As primary eye care providers, we should alert those vulnerable patients to the possibility of vitamin D deficiency. Vitamin D may have a role to play in preventing and treating systemic and ocular conditions such as age-related macular degeneration and diabetic retinopathy. We should also promote eye protection and vitamin D supplementation and not regular sun exposure as it may increase a person's risk to skin cancer. Black people absorb more UVB in the melanin of their skin than do White people and, therefore, require more sun exposure to produce the same amount of vitamin D.

Historically, vitamin D was known to be relevant for treating rickets, but recently accumulated data suggests that it might also play a role in various extra-skeletal diseases, including $\mathrm{AMD}$, diabetic retinopathy, cardiovascular disease, autoimmune disease, certain types of cancers, infectious disease and neuropsychological conditions. The latest research suggests that sunlight may be more essential for health then was previously thought.

\section{Acknowledgements}

I would like to thank Professor WF Harris for encouraging me to research this topic and have my vitamin D status tested, which, as he predicted, was found to be low.

\section{Competing interests}

The author declares that he has no financial or personal relationship(s) which may have inappropriately influenced him in writing this article.

\section{References}

1. Holick MF. Vitamin D. Evolutionary, physiological and health perspectives. Curr Drug Targets. 2011;12(1):4-18. http://dx.doi.org/10.2174/138945011793591635

2. Holick MF. The vitamin epidemic and its health consequences. J Nutr. 2005;135(11):2739S-2748S

3. Holick MF, Chen TC. Vitamin D deficiency: A worldwide problem with health consequences. Am J Clin Nutr. 2008;87(4):1080S-1086S.

4. Lips P. Vitamin D physiology. Prog Biophys Mol Biol. 2006;92(1):4-8. http://dx.doi. org/10.1016/j.pbiomolbio.2006.02.016

5. Pelino CJ, Pizzimenti JJ. Vitamin D comes to light. Rev Optom. 2013;Nov:78-79.

6. Holick MF. Sunlight and vitamin D for bone health and prevention of autoimmune diseases, cancer and cardiovascular disease. Am J Clin Nutr. 2004;80(6 Suppl):1678S-1688S.

7. Holick MF. Vitamin D deficiency. N Engl J Med. 2007;357(3):266-281. http:// dx.doi.org/10.1056/NEJMra070553

8. Holick MF. Vitamin D: A millennium perspective. J Cell Biochem. 2003;88(2):296307. $h$ ttp://dx.doi.org/10.1002/jcb.10338

9. Berridge MJ. Calcium signalling remodelling and disease. Biochem Soc Trans. 2012;40(2):297-309. http://dx.doi.org/10.1042/BST20110766

10. D'Orazio J, Jarrett S, Amaro-Ortiz A, Scott T. UV radiation and the skin. Int J Mol Sci. 2013;14(6):12222-122248. http://dx.doi.org/10.3390/ijms140612222

11. Bendik I, Friedel A, Ross FF, Weber P, Eggerdorfer M. Vitamin D: A critical and essential micronutrient for human health. Frontiers Physiol. 2014;5(7):1-14. http://dx.doi.org/10.3389/fphys.2014.00248

12. Holick MF. McCollum Award Lecture, 1994: Vitamin D: New horizon for the 21st century. Am J Clin Nutr. 1994;60(4):619-630.

13. Holick MF. Vitamin D: Its role in cancer prevention and treatment. Prog Biophys Mol Biol. 2006;92(1):49-59. http://dx.doi.org/10.1016/j.pbiomolbio.2006.02.014

14. Mathieu C, Gysemans C, Giulietti A, Bouillon R. Vitamin D and diabetes. Diabetologia. 2005;48(7):1247-1257. http://dx.doi.org/10.1007/s00125-0051802-7

15. Holick MF. Diabetes and the vitamin D connection. Curr Diabet Reports. 2008;8(5):393-398. http://dx.doi.org/10.1007/s11892-008-0068-0

16. Parekh N, Chappell RJ, Millen AE, Albert DM, Mores JA. Association between vitamin $\mathrm{D}$ and age-related macular degeneration in the Third National Health and Nutrition Examination Survey, 1988 through 1994. Arch Ophthalmol. 2007;125(5):661-669. http://dx.doi.org/10.1001/archopht.125.5.661

17. Timms PM, Mannan N, Hitman GA. Circulating MMP9, vitamin D and variation in the TIMP-1 response with VDR genotype: Mechanisms for inflammatory damage in chronic disorders? QJM. 2002;95(12):787-796. http://dx.doi.org/10.1093/ qjmed/95.12.787

18. Millen $A E$, Voland $R$, Sondel $S A$, et al. Vitamin $D$ status and early age-related macular degeneration in postmenopausal women. Arch Ophthalmol. 2011;129(4):481-489. http://dx.doi.org/10.1001/archophthalmol.2011.48

19. Kaur H, Donaghue KC, Chan AK, et al. Vitamin D deficiency is associated with retinopathy in children and adolescents with type 1 diabetes. Diabetes Care. 2011;34(6):1400-1402. http://dx.doi.org/10.2337/dc11-0103

20. Seddon JM, Reynolds R, Shah HR, Rosner B. Smoking, dietry, betain, methionine, and vitamin $\mathrm{D}$ in monozygomatic twins with discordant macular degeneration epigenetic implication. Ophthalmol. 2011;118(7):1386-1394.

21. Fjeldastad C, Fjeldastad AS, Weir JP, Pardo G. Association of vitamin D deficiency with RNFL thickness in MS individuals without history of optic neuritis. Mult Scler Relat Disord. 2014;3(4):489-493. http://dx.doi.org/10.1016/j. msard.2014.03.001 
22. Mutti DO, Marks AR. Blood levels of vitamin D in teens and young adults with myopia. Optom Vis Sci. 2011;88(3):377-382. http://dx.doi.org/10.1097/ OPX.0b013e31820b0385

23. Snow KK, Seddon JM. Do age-related macular degeneration and cardiovascular disease share common antecedents? Ophthalmic Epidemiol. 1996;6(2):125-143. http://dx.doi.org/10.1076/opep.6.2.125.1558

24. Friedman E. The role of the atherosclerotic process in the pathogenesis of age-related macular degeneration. Am J Ophthalmol 2000;130(5):685-663. http://dx.doi.org/10.1016/S0002-9394(00)00643-7

25. Sun C, Klein R, Wong TY. Age-related macular degeneration and the risk of coronary heart disease and stroke. Ophthalmol. 2009;116(10):1913-1919. http:// dx.doi.org/10.1016/j.ophtha.2009.03.046

26. Fernandez $A B$, Wong $T Y$, Klein $B$, et al. Age-related macular degeneration and incident cardiovascular disease: The multi-ethnic study of antherosclerosis. Ophthalmol. 2012;119(4):765-770. http://dx.doi.org/10.1016/j.ophtha.2011.09.044

27. Mullin GC, Dobs A. Vitamin D and its role in cancer and immunity: A prescription for sunlight. Nutr Clin Pract. 2007;22(3):305-322. http://dx.doi. org/10.1177/0115426507022003305

28. Mascitelli L, Pezzetta F, Goldstein MR. Macular degeneration, heart disease, and vitamin D. Ophthalmol. 2010;117(1):194. http://dx.doi.org/10.1016/j. ophtha.2009.09.048

29. Patrick PA, Visintainer PF, Shi Q, Weiss IA, Brand DA. Vitamin D and retinopathy in adults with diabetes mellitus. Arch Ophthalmol. 2012;130(6):756-760. http:// dx.doi.org/10.1001/archophthalmol.2011.2749

30. Shimo N, Yasuda T, Kaneto H. Vitamin D deficiency is significantly associated with retinopathy in young Japanese type 1 diabetic patients. Diabetes Res Clin Pract. 2014;106(2):e41-e43. http://dx.doi.org/10.1016/j.diabres.2014.08.005

31. Payne JF, Ray R, Watson DG, et al. Vitamin $D$ insufficiency in diabetic retinopathy. Endocr Pract. 2012;18(2):185-193. http://dx.doi.org/10.4158/EP11147.OR
32. Annwiler C, Milea D, Beauchet O. Dietary vitamin D and age-related macular degeneration. Ophthalmol. 2012;119(5):1090.

33. Murphy A, Kelley B, Nyame YA, et al. Predictors of serum vitamin D levels in African American and European American men in Chicago. Am J Mens Health 2012;6(5):420-426. http://dx.doi.org/10.1177/1557988312437240

34. Benjamin A, Moriakova A, Akhter N, et al. Determinants of 25 -hydroxyvitamin D levels in African-American and Caucasian male veterans. Osteoporos Int. 2009; 20(10):1795-1803. http://dx.doi.org/10.1007/s00198-009-0873-6

35. Annweiler C, Souberbielle JC, Schott AM, de Decker L, Berrut G, Beauchet O. Vitamin D in the elderly: 5 points to remember. Geriatr Psychol Neuropsychiatr Vieil. 2011;9(3):295-267.

36. Ritu G, Gupta A. Vitamin D deficiency in India: Prevalence, causalities and interventions. Nutrients. 2014;6(2):729-775. http://dx.doi.org/10.3390/nu6020729

37. Rosen C. Vitamin D insufficiency. N Engl J Med. 2011;364(3):248-254. http:// dx.doi.org/10.1056/NEJMcp1009570

38. Harris SS. Vitamin D and African American. J Nutr. 2006;136(4):1126-1129.

39. Nair R, Maseeh A. Vitamin D: The sunshine vitamin. J Pharmacol Pharmacother 2012;3(2):118-126.

40. Buyukuslu N, Esin K, Hizli H, Sunal N, Yigit P, Garipagaoglu M. Clothing preference affects vitamin D status of young women. Nutr Res. 2014;34(8):688-693. http:// dx.doi.org/10.1016/j.nutres.2014.07.012

41. Varsavsky M, Alonso G, Garcia-Martin A. Vitamin D: Present and future. Rev Clin Esp. 2014;214(7):396-402. http://dx.doi.org/10.1016/j.rce.2014.04.003

42. Heaney RP. Vitamin D in health and disease. Clin J Am Soc Nephrol. 2008;3(5): 1535-1541. http://dx.doi.org/10.2215/CJN.01160308

43. Jacobs $\mathrm{CH}$, Holick $M F$, Shao $Q$, et al. Hypervitaminosis $D$ associated with drinking milk. N Engl J Med. 1992;326(18):1173-1177. http://dx.doi.org/10.1056/ NEJM199204303261801 